\title{
Does obesity hinder radiotherapy in endometrial cancer patients? The implementation of new techniques in adjuvant radiotherapy - focus on obese patients
}

\author{
Małgorzata Moszyńska-Zielińska ${ }^{1}$, Justyna Chałubińska-Fendler², Leszek Gottwald ${ }^{1,2}$, \\ Leszek Żytko ${ }^{1}$, Ewelina Bigos ${ }^{1}$, Jacek Fijuth ${ }^{1,2}$ \\ ${ }^{1}$ Teleradiotherapy Department, Regional Oncological Centre, Lodz, Poland \\ ${ }^{2}$ Radiotherapy Department, Chair of Oncology, Medical University of Lodz, Poland
}

\begin{abstract}
The increasing incidence of obesity in Poland and its relation to endometrioid endometrial cancer (EEC) is resulting in the increasing necessity of treating obese women. Treatment of an overweight patient with EEC may impede not only the surgical procedures but also radiotherapy, especially external beam radiotherapy (EBRT). The problems arise both during treatment planning and when delivering each fraction due to the difficulty of positioning such a patient - it implies the danger of underdosing targets and overdosing organs at risk. Willingness to use dynamic techniques in radiation oncology has increased for patients with EEC, even those who are obese. During EBRT careful daily verification is necessary for both safety and treatment accuracy. The most accurate method of verification is cone beam computed tomography (CBCT) with soft tissue assessment, although it is time consuming and often requires a radiation oncologist. In order to improve the quality of such treatment, the authors present the practical aspects of planning and treatment itself by means of dynamic techniques in EBRT. The authors indicate the advantages and disadvantages of different types of on-board imaging (OBI) verification images. Considering the scanty amount of literature in this field, it is necessary to conduct further research in order to highlight proper planning and treatment of obese endometrial cancer patients. The review of the literature shows that all centres that wish to use EBRT for gynaecological tumours should develop their own protocols on qualification, planning the treatment and methods of verifying the patients' positioning.
\end{abstract}

Key words: obesity, endometrial cancer, radiotherapy.

\section{Introduction}

According to data derived from the Polish National Cancer Registry, in the year 2010 endometrioid endometrial cancer (EEC) is the most common malignant tumour of the female genitourinary system [1]. Its increasing morbidity is linked to better life conditions, longer life expectancy, changes in life style and nutrition habits [2]. One of the major risk factors for developing EEC is obesity, which has been a growing problem across Poland in recent years [2-6]. An important aspect associated with obesity is decreased quality of life reported by obese patients with EEC [7]. However, obesity not only reduces quality of life and impedes surgical procedures performed on the patients, but also complicates adjuvant radiotherapy [8]. During radiotherapy of patients with EEC, particularly those with obesity, positioning errors are more likely to exceed margins which were taken into consideration during planning [9]. Modern radiotherapy techniques, including dynamic dose administration, as well as the verification of patient's positioning, may be helpful in reducing the dose in organs at risk (OARs), enabling the appropriate dose distribution in targets $[10,11]$. However, equipment needed for on-board imaging (OBI) verification on a daily basis is unfortunately not available in all radiotherapy departments in Poland.

The aim of this review is to present the possibilities of solving problems with radiotherapy of obese EEC patients. Given the single-centre experience of the authors from the Regional Oncological Centre in Copernicus Memorial Hospital in Łódź, the paper will focus on the aspect of modern external beam radiotherapy (EBRT). 
Rules to be followed when qualifying an obese endometrioid endometrial cancer patient for external beam radiotherapy in our centre

It is indicated to use EBRT after primary surgery for all EEC except for those staged IV, IA or G1/G2. External beam radiotherapy is generally used after the patient has undergone the full surgical and pathologic protocol: panhysterectomy including adnexectomy, pelvic lymphadenectomy and, if needed, para-aortic lymphadenectomy. External beam radiotherapy reduces the risk of local failure and prolongs overall survival. Patients with high risk of recurrence benefit from EBRT the most. Another indication, however rare, is primary EBRT for EEC in patients who had no prior surgery. This situation occurs when there are contraindications to surgery, usually due to significant co-morbidities. Also patients with EEC with local recurrence after no previous radiotherapy may be considered candidates for EBRT [12-15].

In our department we treat over 3600 patients a year, including 170 patients with EEC. The first step to qualify the patient for EBRT is a meeting with consultants of radiation oncology and gynaecology. During that meeting the initial qualification (or disqualification) is performed and therapeutic targets, doses and radiation techniques are selected. The final qualification is done at a department meeting. Before the treatment starts, a computed tomography (CT) scan with a contrast agent of the abdomen and pelvis is performed for planning purposes. For the $\mathrm{CT}$ procedure, the patient is set up in a position that must be accurately replicated during treatment. Following the $\mathrm{CT}$ scan, the radiation oncologist contours several types of targets: gross, clinical and planned target volumes (GTV, CTV and PTV respectively). For each target volume, the prescribed radiation dose is subsequently calculated by a medical physicist. At this stage, OARs are also contoured as recommended by the ICRU report (International Commission on Radiation Units and Measurements) [16]. Patients are usu-

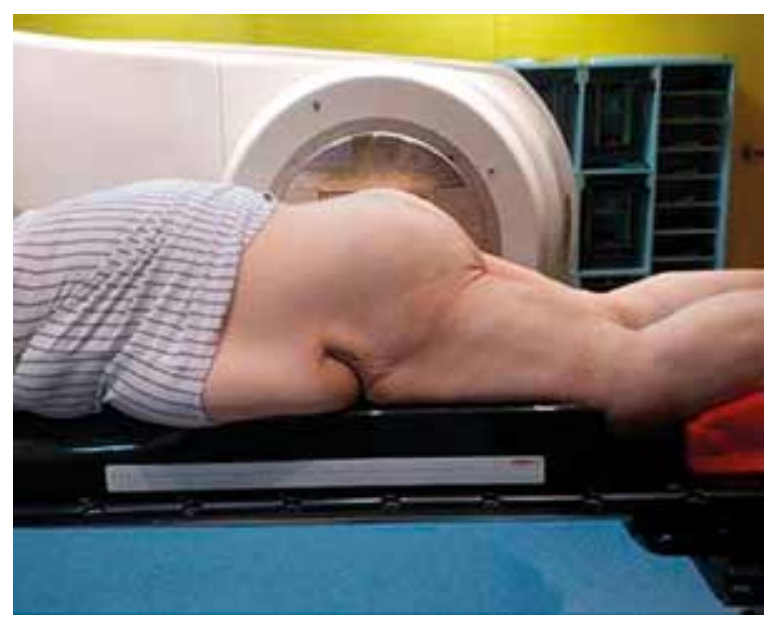

Fig. 1. Prone position with use of the BellyBoard ally positioned supine with sponge blocks placed under the calves. Small permanent dots are tattooed on both sides above the trochanters and on the centre of the abdomen. Due to problems with positioning of obese patients a BellyBoard is used and patients are set up prone (Fig. 1) as in the supine position it would be impossible to align the tattoos due to excessive fat tissue on the abdomen. The BellyBoard thus allows the patient to stay in a much more stable position [17]. To ensure the proper and repeatable position of the patient, the tattoo marker in such cases is located on the patient's back in the lumbar area. Another possibility of proper positioning of obese patients is to use vacuum mattresses.

Contouring is performed after acquiring the $\mathrm{CT}$ scans of a properly positioned patient. The OARs in the pelvis include the rectum, small intestine, urinary bladder, spinal cord and the femoral heads. The contoured GTV is a visible surgical scar inside the pelvis. The CTV covers the GTV regions and regional lymph nodes (Fig. 2). In obese patients due to excessive fat tissue, artefacts may appear during image acquisition and may thus hinder contouring of both targets and OARs [11]. In general, the PTV is the CTV enlarged to account for set-up errors and breathing mobility of the organs by adding $0.5-1.0 \mathrm{~cm}$.

\section{External beam radiotherapy fechniques in patients with endometrioid endometrial cancer - advantages and disadvantages}

Before the era of dynamic techniques, patients with EEC were irradiated using the 4-static field technique. This provided a homogeneous dose distribution in the whole target so the 3-5 $\mathrm{mm}$ set-up errors would not result in underdosing of the targets (PTV, CTV). Consequently, the doses in OARs were unnecessarily high, in contrast to doses planned with dynamic techniques (Fig. 3) [10].

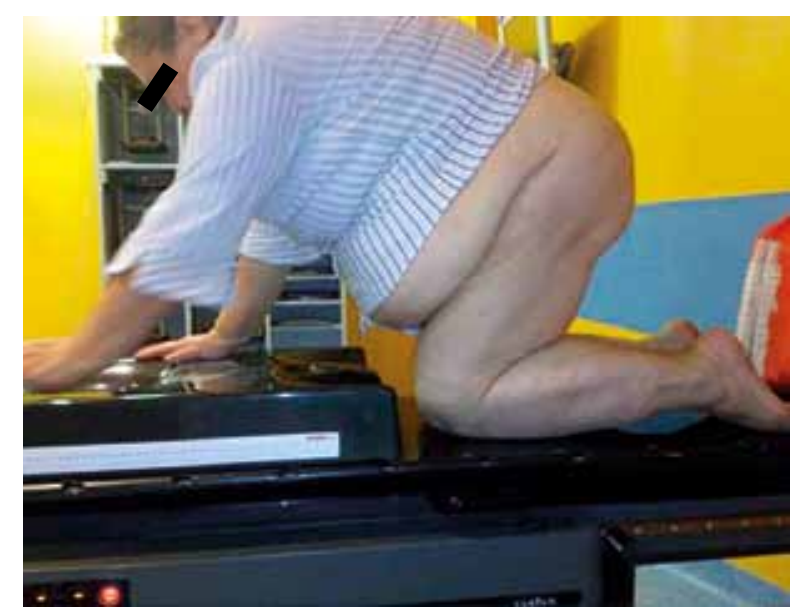



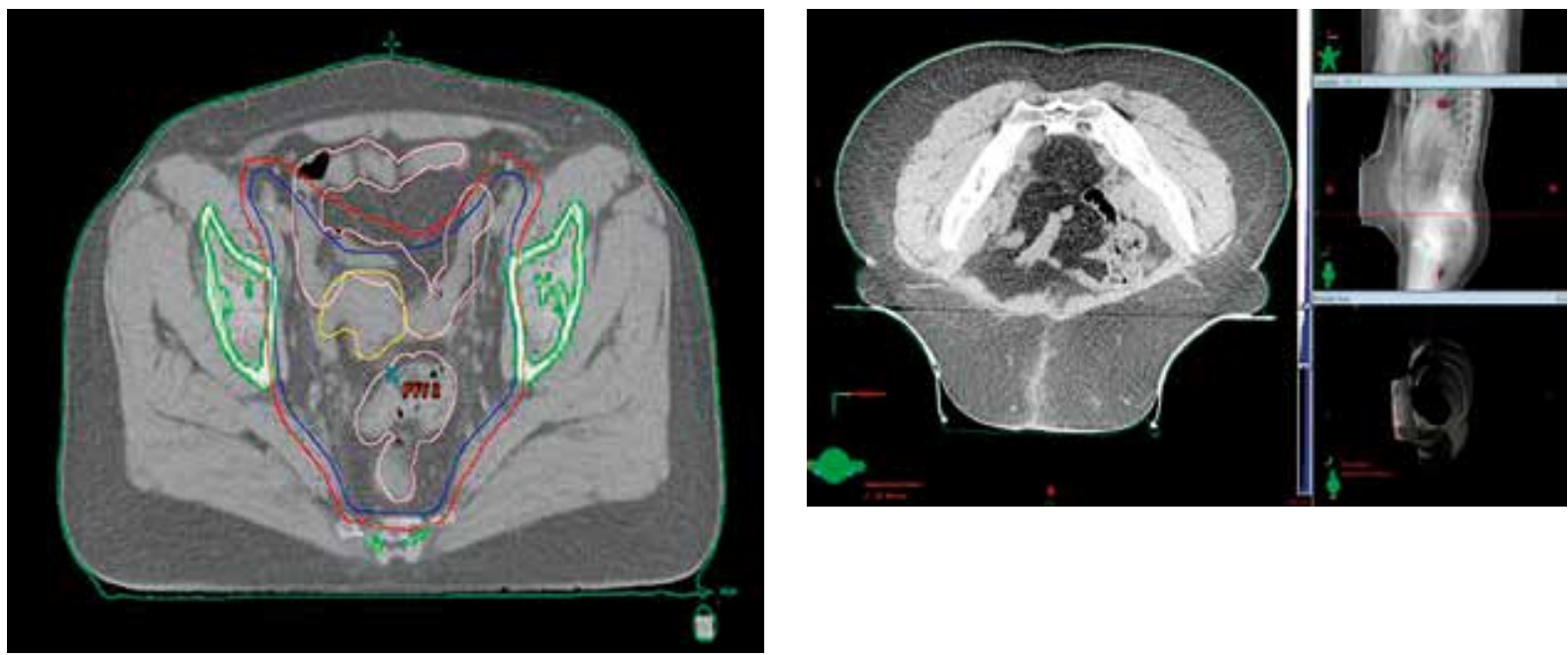

Fig. 2. Scans from a planning CT. The patient is positioned on a BellyBoard. Visible contours of targets: Blue line - CTV, red line - PTV
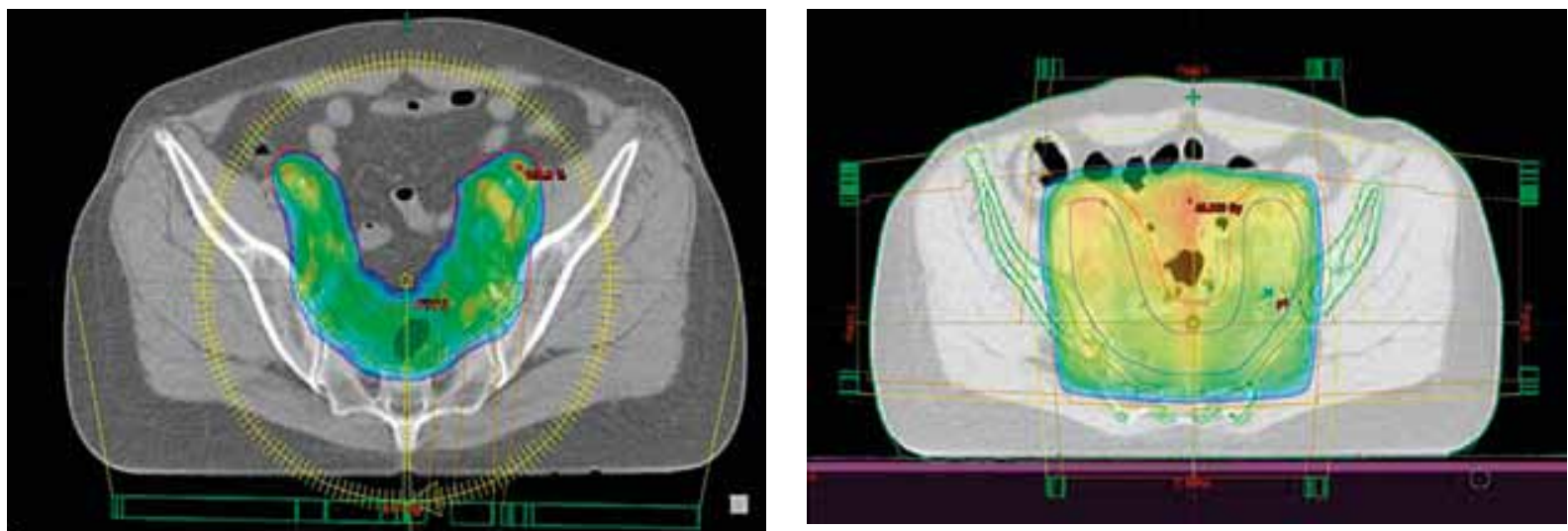

Fig. 3. External beam planning assessment - colour wash presents prescribed dose distribution. Left side shows the VMAT technique with high dose planned only for the target. The right side shows the 4 static fields - called the box technique - with the high dose shown not only for the target but also other regions

Three-dimensional conformal IMRT (intensity-modulated radiation therapy) techniques were first developed in order to decrease the doses in OARs. It soon became apparent that they also allowed for improved dose optimization in target volumes, which is popularly called "dose painting". Further development of IMRT techniques by developing VMAT (volumetric arc therapy) additionally allowed treatment time of each fraction to be reduced. The downside of dynamic techniques is that their increased accuracy makes them more prone to malpositioning errors. Hence, moving out from the target by as little as 2-3 $\mathrm{mm}$ may result in an underdosage in the target and/or a simultaneous rapid increase of the dose received by OARs. Therefore, the cornerstone of dynamic technique procedures in EBRT is accurate verification of the patient's position on a daily basis.

\section{Verification of the patient's position}

Most modern linear accelerators (e.g. Clinac 2300, Varian) are equipped with kilovoltage (KV) X-ray imag- ing tools, which allow daily verification to be performed. Images in at least two projections - coronal and sagittal - must be used to assess the bone structures on the KV image. These are compared to the patient's baseline position established during the initial CT (Fig. 4A). The patient's position may also be verified by means of cone beam CT (CBCT), whose images are derived from the same X-ray lamp producing KV images and compared to the patient's initial position. The advantage of CBCT over KV images is the fact that it allows the technician or attending doctor to align the patient's position not only by using bone structure but also using soft tissues for reference. Cone beam CT verification may however require a radiation oncologist's consultation. On the other hand, the KV images are acquired faster and can be assessed easily by a radiation technician, speeding up the process. Automated verification systems exist (e.g. Exac Trac) and may be used to verify the patient's position by producing multidimensional, sometimes difficult to evaluate, images. Verification of the patient's position is necessary and should be opti- 


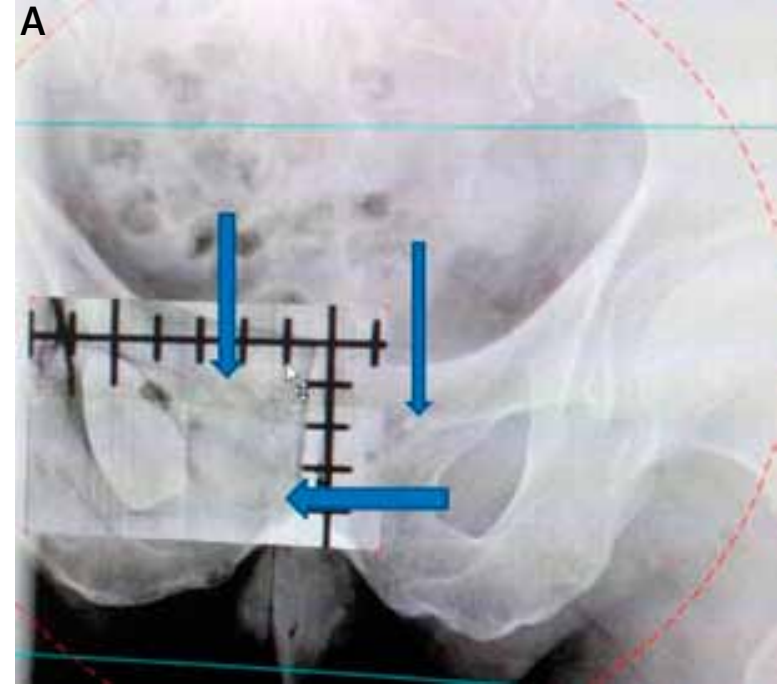

Fig. 4. Verification of the patient's position. A) Using a KV image - coronal view - floating window represents actual position which is compared to the position on the image derived from planning $\mathrm{CT}$. Arrows indicate the deviations in positioning. B) Using CBCT image - coronal and sagittal view - floating window represents actual position on the $\mathrm{CBCT}$ which is compared to the position on the image derived from the planning CT

mally performed on a daily basis when using IMRT or VMAT. Daily assessment of the patient's positioning provides the staff with information about the size of positioning errors, which are crucial to set own protocols for PTV size. According to the literature, the patient's weight has a significant impact on error size, even up to $7 \mathrm{~mm}$ observed in nearly $30 \%$ of obese patients with EEC [11].

\section{Summary}

In treating patients with EEC one must consider that not only the primary diagnosis affects the mode of treatment and resulting prognosis. Additional clinical factors, such as obesity, may have a high impact on the possibility of performing and efficacy of surgery and adjuvant EBRT. A BMI above $30 \mathrm{~kg} / \mathrm{m}^{2}$ seems to have a significant impact on the rate of malpositioning and size of positioning errors, which may force radiation oncologists to adopt bigger margins for PTV during EBRT planning.

Willingness to use dynamic techniques in radiation oncology has increased for patients with EEC, even those who are obese. During EBRT careful daily verifica-
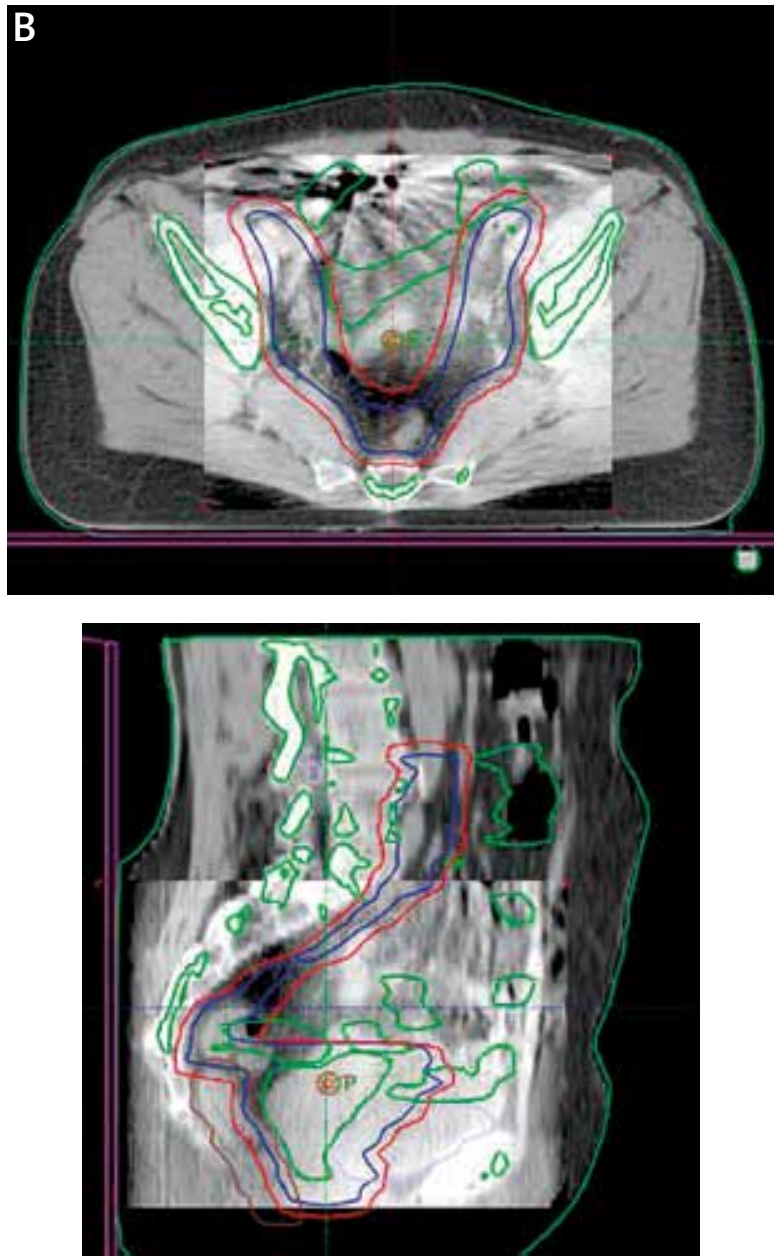

tion is necessary for both safety and treatment accuracy. The most accurate method of verification is $\mathrm{CBCT}$ with soft tissue assessment, although it is time consuming and often requires a radiation oncologist.

The literature on IMRT and VMAT mostly focuses on prostate or head and neck cancer patients. The number of articles on IMRT or VMAT used in patients with EEC is still very low. Considering the facts mentioned above, all centres that wish to use EBRT for gynaecological tumours should develop their own protocols on qualification, planning the treatment and methods of verifying the patients' positioning.

\section{Disclosure}

Authors report no conflicts of interest.

\section{References}

1. Zakład Epidemiologii i Prewencji Nowotworów Centrum Onkologii Instytut w Warszawie. Krajowa baza danych nowotworowych. http:// www.onkologia.org.pl.

2. Fader AN, Arriba LN, Frasure HE, et al. Endometrial cancer and obesity: epidemiology, biomarkers, prevention and survivorship. Gynecol Oncol 2009; 114: 121-127. 
3. Jodkowska M, Oblacinska A, Tabak I. Overweight and obesity among adolescents in Poland: gender and regional differences. Public Health Nutr 2010; 13: 1688-1692.

4. Mackintosh M, Crosbie E. Obesity-driven endometrial cancer: is weight loss the answer? BJOG 2013; 120: 791-794.

5. Richardson LC, Thomas C, Bowman BA. Obesity and endometrial cancer: challenges for public health action. Womens Health (Lond Engl) 2009; 5: 595-597.

6. Arem H, Irwin ML. Obesity and endometrial cancer survival: a systematic review. Int J Obes (Lond) 2013; 37: 634-639.

7. Fader AN, Frasure HE, Gil KM, et al. Quality of life in endometrial cancer survivors: what does obesity have to do with it? Obstet Gynecol Int 2011; 2011: 308609.

8. Martra F, Kunos C, Gibbons H, et al. Adjuvant treatment and survival in obese women with endometrial cancer: an international collaborative study. Am J Obstet Gynecol 2008; 198: 89.e1-8.

9. Harris EE, Latifi K, Rusthoven C, et al. Assessment of organ motion in postoperative endometrial and cervical cancer patients treated with intensity-modulated radiation therapy. Int J Radiat Oncol Biol Phys 2011; 81: e645-650.

10. Chan P, Yeo I, Perkins G, et al. Dosimetric comparison of intensity-modulated, conformal, and four-field pelvic radiotherapy boost plans for gynecologic cancer: a retrospective planning study. Radiat Oncol 2006; 1: 13
11. Lin LL, Hertan L, Rengan R, et al. Effect of body mass index on magnitude of setup errors in patients treated with adjuvant radiotherapy for endometrial cancer with daily image guidance. Int J Radiat Oncol Biol Phys 2012; 83: 670-675.

12. Benedet JL, Bender $\mathrm{H}$, Jones $\mathrm{H}$, 3rd, et al. FIGO staging classifications and clinical practice guidelines in the management of gynecologic cancers. FIGO Committee on Gynecologic Oncology. Int J Gynaecol Obstet 2000; 70: 209-262.

13. De Sanctis V, Agolli L, Valeriani M, et al. External-beam radiotherapy and/or HDR brachytherapy in postoperative endometrial cancer patients: clinical outcomes and toxicity rates. Radiol Med 2013; 118: 311 322.

14. Nout RA, Smit VT, Putter H, et al. Vaginal brachytherapy versus pelvic external beam radiotherapy for patients with endometrial cancer of high-intermediate risk (PORTEC-2): an open-label, non-inferiority, randomised trial. Lancet 2010; 375: 816-823.

15. Inciura A, Atkocius V, Juozaityte E, et al. Long-term results of high-doserate brachytherapy and external-beam radiotherapy in the primary treatment of endometrial cancer. J Radiat Res 2010; 51: 675-681.

16. Chavaudra J, Bridier A. Definition of volumes in external radiotherapy: ICRU reports 50 and 62. Cancer Radiother 2001; 5: 472-478.

17. Pinkawa M, Gagel B, Demirel C, et al. Dose-volume histogram evaluation of prone and supine patient position in external beam radiotherapy for cervical and endometrial cancer. Radiother Oncol 2003; 69: 99-105. 